KYUSHU-HET-95

\title{
Anomalous dimensions determine the power counting - Wilsonian RG analysis of nuclear EFT -
}

\author{
Koji Haradat and Hirofumi Kubd \\ Department of Physics, Kyushu University \\ Fukuoka 812-8581 Japan
}

(Dated: August 31, 2018)

\begin{abstract}
The Legendre flow equation, a version of exact Wilsonian renormalization group (WRG) equation, is employed to consider the power counting issues in Nuclear Effective Field Theory. A WRG approach is an ideal framework because it is nonperturbative and does not require any prescribed power counting rule. The power counting is determined systematically from the scaling dimensions of the operators at the nontrivial fixed point. The phase structure is emphasized and the inverse of the scattering length, which is identified as a relevant coupling, is shown to play a role of the order parameter. The relations to the work done by Birse, McGovern, and Richardson and to the Kaplan-Savage-Wise scheme are explained.
\end{abstract}

*Electronic address: koji1scp@mbox.nc.kyushu-u.ac.jp

${ }^{\dagger}$ Electronic address: kubo@higgs.phys.kyushu-u.ac.jp 


\section{INTRODUCTION}

Conventional nuclear theory is based on force potentials. There have been much progress and we now have very accurate ones such as Nijmegen 1] , Argonne $V_{18}$ [2], and CD-Bonn [3, 4] potentials which fit nicely to about 3,000 nucleon-nucleon scattering data with energies up to $350 \mathrm{MeV}$. The potential models have matured to the demand of precise numerical calculations.

Potential models however have serious drawbacks. Even though they are very precise, after all, they are just (semi-phenomenological) models. It is not obvious how these models are related to QCD, the fundamental theory of strong interactions, nor how to improve them in a systematic manner. Proper treatment of inelastic processes seems formidable. In addition, the potential approach is known to suffer from the so-called off-shell ambiguities, which may cause serious problems for a correct understanding of multi-nucleon systems.

Nuclear effective field theory (NEFT) [5, 6] is expected to be a promising alternative. It is based on very general principles of quantum field theory [7, 8, 9, 10, 11, 12], and linked to QCD through chiral symmetry, while, instead of quarks and gluons, only the relevant degrees of freedom (nucleons, and optionally pions, deltas, etc.) at low energies are considered. Chiral perturbation theory $(\chi \mathrm{PT})[13$, 14], a prominent example of EFT in which only meson degrees of freedom are treated explicitly, has been applied very successfully. In short, NEFT aims at a similar success.

Since a Lagrangian of EFT contains an infinite number of operators allowed by the symmetry, one needs a power counting rule to give orderings to an infinite number of Feynman diagrams generated by the Lagrangian. Once it is specified, physical quantities can be calculated in a systematic expansion in powers of $Q / \Lambda_{0}$, where $\Lambda_{0}$ is the cutoff, above which the EFT is not valid, and $Q\left(<\Lambda_{0}\right)$ is the typical momentum of the process one is interested in. In the case of perturbative systems such as meson-meson scattering, the power counting which is determined on the basis of naive dimensional analysis(NDA) 15] is known to give correct results. NEFT is, on the other hand, inherently nonperturbative. (There are bound states, the nuclei!) In addition, it is a fine-tuned system; the inverses of the nucleon-nucleon scattering lengths $1 / a^{\left({ }^{1} S_{0}\right)} \sim-8 \mathrm{MeV}$, and $1 / a^{\left({ }^{3} S_{1}\right)} \sim 40 \mathrm{MeV}$ are much smaller than $\Lambda_{0} \sim m_{\pi} \sim 140 \mathrm{MeV}$ for the pionless NEFT. (If we consider the pionful NEFT, $\Lambda_{0}$ should be larger, presumably $\Lambda_{0} \sim 500 \mathrm{MeV}$, and the system should be considered more 
fine-tuned.) Moreover deuteron is a shallow bound state with binding energy of $2.2 \mathrm{MeV}$. We need a power counting scheme which takes into account the "unnaturalness."

In this paper, we consider a systematic way of determining the power counting for such nonperturbative and fine-tuned systems on the basis of Wilsonian RG analysis. As we will explain below, our approach is very general and not restricted only to NEFT.

At present, there are basically two power counting schemes proposed for NEFT. In Weinberg's scheme [5, 6], one identifies the sum of irreducible diagrams in the expansion based on NDA as the "effective potential," and solves the Lippmann-Schwinger (L-S) equation with it. The "effective potential" is iterated to all order, i.e., all operators are treated nonperturbatively. Although conceptually attractive and simple in numerical implementation, Weinberg's power counting has been shown to be inconsistent: the divergences that arise in the leading order calculation cannot be absorbed by the leading order operators in certain partial waves 16, 17]. The other power counting scheme proposed by Kaplan, Savage and Wise (KSW) 18, 19] begins with a renormalization group (RG) behavior at a nontrivial fixed point, which corresponds to a system with infinite scattering length. In contrast to Weinberg's scheme, only the nonderivative contact operator is treated nonperturbatively while others are treated perturbatively. Although the KSW power counting is a consistent scheme in the sense that results are independent of subtraction point at every order of the expansion, serious problems have been reported. Fleming, Mehen, and Stewart (FMS) 20] calculated the nucleon-nucleon scattering amplitudes up to next-to-next-to leading order in a NEFT with pions included ("pionful" NEFT), implementing the KSW power counting, and found that the scattering amplitude in the ${ }^{3} S_{1}-{ }^{3} D_{1}$ channel shows no convergence at all. A possible explanation for the success and the failure has been given in Ref. [21, 22].

The present status may be summarized as that NEFT works "pretty well if one follows a patchwork of power counting rules 23]." Actually, in spite of the problems mentioned above, many of higher order calculations in both power counting schemes have been found to be successful at least numerically. It might be however too early to say that the problems are settled. One should keep in mind that, besides the numerical agreement with data, a thorough understanding of power counting in a systematic and consistent way is extremely important. It is this issue that we address in this paper.

We believe that Wilsonian Renormalization Group(WRG) [24, 25] approach can provide the key. This approach allows us to analyze the behavior of the operators (or the corre- 
sponding coupling constants) by assuming only the symmetry and the relevant degrees of freedom. Most importantly to our present purpose, WRG analysis is nonperturbative and does not require any prescribed power counting scheme.

Our basic assertion is that it is anomalous dimensions that determine the power counting. We are going to show in this paper that it is very natural to determine the power counting of an operator from the corresponding scaling dimension (the sum of the canonical and the anomalous dimensions). Our reasoning is based on the very general idea that the power counting should be determined by dimensional analysis. Furthermore, our WRG approach is applicable both to trivial and nontrivial fixed points; near the trivial fixed point, it coincides with NDA, while it explains why the KSW power counting is successful to some extent near the nontrivial fixed point. The real two-nucleon system happens to be close to the nontrivial fixed point, but the WRG approach has a wider scope.

Wilsonian approach has been applied by Birse, McGovern, and Richardson[26] to nonrelativistic two-body scattering to obtain a power counting rule. They considered a Wilsonian RG equation for an effective potential, and identified two fixed points; a trivial one and a nontrivial one. Then they determined how to organize the terms in the effective potential by considering the linearized RG equation around the fixed point. Although the potential they considered is general, the physical meaning of energy-dependence of it in the Schrödinger equation is obscure. Furthermore it does not seem to be very clear how the symmetry of the theory is implemented in the potentials. Since this potential approach is similar to traditional potential model approach, it might share some of their problems. It is desirable to understand the power counting in a completely field theoretical framework to benefit from all the good features of EFT. The relation between their work and the present work will be explained in Sec. $\mathbf{\nabla}$ in detail.

A WRG analysis in field theory requires all possible operators consistent with the assumed symmetry. In the previous paper [27], we emphasized the role played by "redundant operators," which may be eliminated by field redefinitions. We then explicitly showed that the nonperturbative off-shell amplitude can be renormalized only by properly treating redundant operators in the WRG analysis. However, the method of the calculation is rather straightforward and seems applicable only for simple cases. A more powerful method is needed for general cases.

In this paper we study the RG flow for the pionless NEFT employing Legendre flow 
equation, which is one of the implementations of Wilsonian RG. We obtain the RG flows for ${ }^{1} S_{0}$ and ${ }^{3} S_{1}-^{3} D_{1}$ channels to order $\mathcal{O}\left(p^{2}\right)$. We identify two phases in our RG flow for both channels; the strong coupling phase and the weak coupling phase. We also show that the nontrivial fixed point is on the phase boundary of our RG flow. The scaling dimension of the operators around the fixed points are calculated. We find that the anomalous dimension of the operators around the nontrivial fixed point is large. We argue that the scattering length is identified with the order parameter which characterizes these phases.

The pionless NEFT is an interesting theory as an application of nonperturbative RG equation, which is, in most cases, very difficult to treat. Thanks to the nonrelativistic feature of the theory, it allows us to solve (approximately) with a simple truncation of the space of operators, though the justification of the truncation only comes from actually enlarging the space. This theory also provides a simple example of nontrivial fixed point and the existence of a bound state. We hope that analyzing this theory gives some insight into the use of nonperturbative RG to get the information about bound states.

This paper is organized as follows: in Sec. III, we explain why the scaling dimensions are important in determining power counting. The Legendre flow equation for nonrelativistic systems is introduced in Sec III. We present the detailed analysis of pionless NEFT and its physical implications in Sec. IV] The relation to the work done by Birse et al. is clarified and comments on the other schemes, especially on the KSW scheme, are made in Sec. D. Finally in Sec. VI, we summarize our study, and discuss future prospects. Appendix @ provides some technical information about the cutoff function used in the Legendre flow equation. In Appendix $\mathbb{B}$ we derive the RG equations from the two-nucleon scattering amplitudes.

\section{POWER COUNTING AND RENORMALIZATION GROUP}

In this section, we consider what power counting is and its relation to renormalization group in general terms.

The most basic idea behind power counting is the order of magnitude estimate based on dimensional analysis. The order of magnitude of a physical quantity may be estimated by an appropriate combination of dimensionful parameters of typical scales. The period $T$ of a simple pendulum with length $L$ and mass $M$ in a uniform gravitational field with the 
gravitational acceleration $g$ is estimated as $T \sim \sqrt{L / g}$. The dimensionless coefficient is expected to be of order one, the idea of "naturalness." (In the pendulum example, it is a bit large, $2 \pi$.)

There is only one important scale in a simplest (relativistic) field theory; the physical cutoff $\Lambda_{0}$. The mass $m$ is related to $\Lambda_{0}$ by a very large dimensionless constant $\xi$ as $m=\Lambda_{0} / \xi$. (This is a necessary fine-tuning to have a sensible quantum field theory.) Any quantities may be measured in units of $\Lambda_{0}$. The interaction Lagrangian in spacetime dimension $D$ may be written as an infinite sum of the interactions of the form

$$
\mathcal{L}_{i n t}=-\sum_{i} \frac{g_{0 i}}{\Lambda_{0}^{d_{i}-D}} \mathcal{O}_{i}(x)
$$

where $d_{i}$ is the (canonical) dimension of the operator $\mathcal{O}_{i}(x)$ and $g_{0 i}$ is the (bare) dimensionless coupling constant. (It is sometimes useful to say that the (dimensionful) coupling $\tilde{g}_{0 i} \equiv$ $g_{0 i} / \Lambda_{0}^{d_{i}-D}$ has dimension $D-d_{i}$.) Classically (and near the trivial fixed point, see below) this dependence on $\Lambda_{0}$ determines how large the contribution of $\mathcal{O}_{i}$ is. One may expand the contribution in powers of $\left(Q / \Lambda_{0}\right)$, where $Q$ is the typical energy/momentum scale of interest, according to the canonical dimensions of the operators. This is a common situation, and, with a twist of chiral symmetry, true for $\chi \mathrm{PT}^{1}$. The so-called NDA applies to such cases. In some cases, however, quantum fluctuations change the situation drastically.

In a WRG analysis, the cutoff is lowered to $\Lambda<\Lambda_{0}$ by integrating out the fluctuations with momentum $\Lambda<p<\Lambda_{0}$, and thus the interaction Lagrangian may be replaced by

$$
\mathcal{L}_{i n t}=-\sum_{i} \frac{g_{i}(\Lambda)}{\Lambda^{d_{i}-D}} \mathcal{O}_{i}(x) .
$$

The coupling $g_{i}(\Lambda)$ now depends on the "floating" cutoff $\Lambda$ so that the physical quantities do not depend on $\Lambda$. The behavior of $g_{i}(\Lambda)$ contains information about how the quantum fluctuation modifies the importance of the operator $\mathcal{O}_{i}$. The differential equations,

$$
\frac{d g_{i}}{d t}=\beta_{i}(g), \quad t \equiv \ln \left(\frac{\Lambda_{0}}{\Lambda}\right),
$$

\footnotetext{
${ }^{1}$ In $\chi$ PT, the masses of the Nambu-Goldstone bosons play a special role as symmetry breaking parameters, which are not related to the cutoff $\Lambda_{0}$. It is not trivial how to treat them. The standard way is to treat $m^{2} \sim Q^{2}$, which is necessary to be consistent with the meson pole structure of the amplitudes 13 . In the pionless NEFT, which we consider in this paper, there is no such a symmetry breaking parameter (we ignore isospin breaking), and thus no subtlety associated to it.
} 
are called $R G$ equation, and determine the $R G$ flow in the space of all coupling constants.

An important concept in the RG analysis is a fixed point of the $\mathrm{RG}$ flow, which is a solution of $\beta_{i}\left(g^{\star}\right)=0$ for all $i$, where the coupling constants stop running. Suppose we are interested in the behavior of a theory close to a fixed point. The behavior of the RG flow near a fixed point can be examined by considering the linearized RG equation obtained by substituting $g_{i}=g_{i}^{\star}+\delta g_{i}$ into the RG equation keeping only the linear terms in $\delta g_{i}$,

$$
\frac{d}{d t} \delta g_{i}=A_{i j}\left(g^{\star}\right) \delta g_{j}
$$

where $\left.A_{i j}\left(g^{\star}\right) \equiv\left(\partial \beta_{i} / \partial g_{j}\right)\right|_{g^{\star}}$. By diagonalizing $A_{i j}\left(g^{\star}\right)$, we have

$$
\frac{d \boldsymbol{u}_{i}}{d t}=\nu_{i} \boldsymbol{u}_{i}
$$

where $\nu_{i}$ is the eigenvalue and $\boldsymbol{u}_{i}$ is the corresponding eigenvector, which may be immediately integrated as,

$$
\boldsymbol{u}_{i}(\Lambda)=\boldsymbol{u}_{i}\left(\Lambda_{0}\right)\left(\frac{\Lambda}{\Lambda_{0}}\right)^{-\nu_{i}}
$$

The exponent of $\Lambda_{0}, \nu_{i}$, is called scaling dimension of the coupling. Depending on the sign of the scaling dimension, interactions are divided into two groups. An operator with its coupling having $\nu_{i}<0$ is called irrelevant. On the other hand, that with $\nu_{i}>0$ is called relevant. If $\nu_{i}=0$, the operator is called marginal. The scaling dimension is the quantum counterpart of the (canonical) dimension. At the trivial fixed point, $g_{i}^{\star}=0$ for all $i$, it agrees with the canonical one, $\nu_{i}=D-d_{i}$, i.e.,

$$
\frac{g_{i}(\Lambda)}{\Lambda^{d_{i}-D}} \sim \frac{g_{0 i}}{\Lambda_{0}^{d_{i}-D}},
$$

while at a nontrivial fixed point it may be quite different. It should be emphasized that the scaling dimensions are not prescribed but determined by the theory (and the fixed point) itself. This is the beauty of the WRG approach.

Let us consider what happens near a nontrivial fixed point more in detail. Suppose that coupling constant $g_{i}(\Lambda)$ is written as

$$
g_{i}(\Lambda) \sim g_{i}^{\star}+\sum_{k} c_{i k}\left(\frac{\Lambda}{\Lambda_{0}}\right)^{-\nu_{k}}
$$

where $c_{i k}$ is a small constant. First of all, it is important to note that the theory is scale invariant at the fixed point. It means that the theory has no typical scale, though it starts 
with the physical cutoff $\Lambda_{0}$. Actually, the coupling $g_{i}(\Lambda) / \Lambda^{d_{i}-D} \sim g_{i}^{\star} / \Lambda^{d_{i}-D}$ is independent of $\Lambda_{0}$. Therefore, the value of the fixed point itself does not contribute to the power counting, because, as we emphasized above, power counting is based on dimensional analysis, but a scale invariant theory does not have a scale! It is the $\Lambda_{0}$ dependent part (the second term in (2.8) ) that contribute to the power counting.

$$
\frac{g_{i}(\Lambda)}{\Lambda^{d_{i}-D}} \sim \frac{g_{i}^{\star}}{\Lambda^{d_{i}-D}}+\sum_{k} c_{i k} \frac{\Lambda_{0}^{\nu_{k}}}{\Lambda^{d_{i}-D+\nu_{k}}} .
$$

From the dependence on $\Lambda_{0}$, one sees that the $k$-th term in the sum behaves like a coupling with dimension $\nu_{k}$.

In the literature, many authors seem to think that the lowest order contact operator in NEFT should be treated "nonperturbatively," because the value of the corresponding coupling constant is large at the nontrivial fixed point (the first term of (2.9)). As we will show in Sec. IV however, it should be treated "nonperturbatively" because it is relevant at the nontrivial fixed point. We believe that this difference is crucial in understanding the power counting for other operators.

Let us summarize what we explained in this section; power counting is in essence the order of magnitude estimate based on dimensional analysis. But the quantum fluctuations change the dimension drastically in some cases. A WRG analysis takes into account the quantum fluctuations in a controlled way, and gives rise to a RG flow, which is characterized by fixed points. Especially near a nontrivial fixed point, the scaling dimension may be quite different from the canonical one. It is therefore natural to consider the power counting based on the quantum scaling dimensions. The important point is that it is not the value of the nontrivial fixed point, but the scaling dimensions that contribute to the power counting.

Finally, we would like to make a comment on the use of momentum cutoff in EFT. Although it might not be widely understood, the momentum cutoff scheme does not break the EFT expansion. (Of course it is much simpler with dimensional regularization.) The loop contributions which (partially) cancel the $\Lambda_{0}$ in the denominator may be absorbed in the renormalized couplings of lower order. Thus, when expressed in terms of renormalized couplings, EFT with momentum cutoff has a sensible expansion as that with dimensional regularization. 


\section{LEGENDRE FLOW EQUATION FOR NON-RELATIVISTIC THEORY}

Legendre flow equation [28, 29, 30], which we use in our analysis, is one of the implementations of WRG equation. It is formulated as a RG equation for the infrared (IR) cutoff effective action called effective average action. In contrast to the conventional effective action in which all the fluctuations are taken into account, the effective average action includes only the quantum fluctuations with momenta larger than some cutoff $\Lambda$. An infinitesimal change of an effective average action with respect to an infinitesimal change in $\Lambda$ is expressed in a differential equation. Starting from an arbitrary bare action at ultraviolet (UV) scale $\Lambda_{0}$, the effective averaged action successively includes the lower momentum fluctuations as the cutoff is lowered, approaches the conventional effective action in the limit of $\Lambda=0$.

In the case of relativistic theory, it is necessary to define the flow equation in Euclidean space because a cutoff must be imposed on all four components of the momenta in order to respect the Lorentz invariance. In our case of nonrelativistic theory, on the other hand, corresponding symmetry is Galilean invariance and rotation symmetry. There is no obvious way of imposing a cutoff maintaining them. It is a rather annoying issue in applying the method to nonrelativistic cases. Fortunately, as we explain in the next section, the correct way of implementing a cutoff is clear in the two-nucleon system. In this section, however, we naively consider the flow equation defined in Minkowski spacetime and impose a cutoff only on three-momenta, though it breaks Galilean invariance.

We begin by defining IR cutoff generating functional for nonrelativistic nucleons with source terms, $\eta^{\dagger}$ and $\eta$. The generating functional for this system is defined in terms of integration over Grassmann variables, $N(x)$, by

$$
\begin{array}{r}
e^{i W_{\Lambda}\left[\eta^{\dagger}, \eta\right]}=\int \mathcal{D} N \mathcal{D} N^{\dagger} e^{i\left(S_{\Lambda_{0}}+N^{\dagger} \cdot R_{\Lambda}^{(1)} \cdot N+\eta^{\dagger} \cdot N-N^{\dagger} \cdot \eta\right)}, \\
N^{\dagger} \cdot R_{\Lambda}^{(1)} \cdot N \equiv \int \frac{d^{4} p}{(2 \pi)^{4}}\left(N^{\dagger}(p)\right)_{A}\left(R_{\Lambda}^{(1)}(p)\right)_{A B}(N(p))_{B},
\end{array}
$$

where $S_{\Lambda_{0}}$ is an arbitrary bare action composed of local operators at physical UV cutoff scale $\Lambda_{0}$. The indices $A$ and $B$ denote spin and isospin collectively. Fourier transform is defined as follows,

$$
N(x) \equiv \int \frac{d^{4} p}{(2 \pi)^{4}} e^{-i p \cdot x} N(p), \quad N^{\dagger}(x) \equiv \int \frac{d^{4} p}{(2 \pi)^{4}} e^{+i p \cdot x} N^{\dagger}(p) .
$$

The function $R_{\Lambda}^{(1)}(p)$ effectively cuts off the IR part of the fluctuations in the integrand so 
that $W_{\Lambda}\left[\eta^{\dagger}, \eta\right]$ contains the effects of only the fluctuations for momenta larger than $\Lambda$ in the coupling constants. In principle, $R_{\Lambda}(p)$ can be an arbitrary function which satisfies the following properties,

$$
\left|R_{\Lambda}^{(1)}\left(\boldsymbol{p}^{2}\right)\right| \rightarrow\left\{\begin{array}{l}
\infty \text { as }(\boldsymbol{p} / \Lambda)^{2} \rightarrow 0 \\
0 \quad \text { as }(\boldsymbol{p} / \Lambda)^{2} \rightarrow \infty
\end{array}\right.
$$

but for definiteness we use the following IR cutoff function in our analysis ${ }^{2}$,

$$
R_{\Lambda}^{(1)}\left(\boldsymbol{p}^{2}\right)=\frac{\boldsymbol{p}^{2}}{2 M}\left[1-\exp \left[\left(\frac{\boldsymbol{p}^{2}}{\Lambda^{2}}\right)^{n}\right]\right]^{-1}
$$

and take the sharp cutoff limit $n \rightarrow \infty$ in most cases. See the next section.

For convenience we introduce the compact notation,

$$
J_{n} \equiv\left(\eta, \eta^{\dagger}\right), \quad U_{n} \equiv\left(N^{\dagger}, N\right), \quad(n=1,2)
$$

and write the generating functional (3.1) as

$$
e^{i W_{\Lambda}[J]}=\int \mathcal{D} U \exp i\left\{S+\frac{1}{2} U_{n}\left(R_{\Lambda}\right)_{n m} U_{m}+J_{n} U_{n}\right\},
$$

where

$$
\left(R_{\Lambda}\right)_{n m} \equiv\left[\begin{array}{cc}
0 & \left(R_{\Lambda}^{(1)}\right)_{A B} \\
\left(R_{\Lambda}^{(2)}\right)_{A B} & 0
\end{array}\right], \quad\left(R_{\Lambda}^{(2)}\right)_{A B} \equiv-\left(R_{\Lambda}^{(1)}\right)_{B A}
$$

The Legendre transform $\tilde{\Gamma}_{\Lambda}[\Phi]$ of $W_{\Lambda}[J]$ may be defined in the standard way by introducing the expectation value of $U_{n}$ in the presence of the source $J_{n}$,

$$
\tilde{\Gamma}_{\Lambda}[\Phi] \equiv W_{\Lambda}[J]-J_{n} \Phi_{n}, \quad \frac{\delta W_{\Lambda}}{\delta J_{n}}=\left\langle U_{n}\right\rangle \equiv \Phi_{n}
$$

where $\Phi_{n} \equiv\left(\psi^{\dagger}, \psi\right)$ has been introduced.

It is more useful to define $\Gamma_{\Lambda}$, an averaged action, as follows,

$$
\Gamma_{\Lambda} \equiv \tilde{\Gamma}_{\Lambda}-\frac{1}{2} \Phi_{n}\left(R_{\Lambda}\right)_{n m} \Phi_{m}
$$

which satisfies the following Legendre flow equation,

$$
\frac{d \Gamma_{\Lambda}}{d \Lambda}=\frac{i}{2} \operatorname{Tr}\left[\frac{d R_{\Lambda}}{d \Lambda}\left(\Gamma_{(2)}+R_{\Lambda}\right)^{-1}\right]
$$

\footnotetext{
${ }^{2}$ In order to cutoff the momentum in an $M$-independent way, we include $M$ in the cutoff function in this form.
} 
where we have introduced the notation $\left(\Gamma_{(2)}\right)_{n m} \equiv \delta^{2} \Gamma_{\Lambda} / \delta \Phi_{n} \delta \Phi_{m}$ and $\operatorname{Tr}$ denotes the integration over momentum and also the trace in the internal space. Although Legendre flow equation resembles a one-loop equation, it contains nonperturbative information through a full propagator with IR cutoff, $\left(\Gamma_{(2)}+R_{\Lambda}\right)^{-1}$.

We introduce a dimensionless parameter $t=\ln \left(\Lambda_{0} / \Lambda\right)$ and $\tilde{\partial}_{t}$ which denotes the derivative with respect to $t$ that acts only on explicit $t$-dependence of the IR cutoff function $R_{\Lambda}$. The flow equation may be written in an even simpler form,

$$
\frac{d \Gamma_{\Lambda}}{d t}=\frac{i}{2} \operatorname{Tr} \tilde{\partial}_{t}\left[\ln \left(\Gamma_{(2)}+R_{\Lambda}\right)\right]
$$

We may further simplify the expression by using the nonrelativistic feature of the theory. For this purpose, let us split $\left(\Gamma_{(2)}+R_{\Lambda}\right)$ into field independent part $\mathcal{P}$ and field dependent part $\mathcal{F}$. $\mathcal{P}$ is the "full propagator", while $\mathcal{F}$ is the sum of the multipoint "vertices" which have two internal lines and $\Phi_{n}$ attached to each of the external legs. The RHS of (3.12) can be written as

$$
\frac{i}{2} \operatorname{Tr} \tilde{\partial}_{t}\left[\ln \mathcal{P}+\left(\mathcal{P}^{-1} \mathcal{F}\right)-\frac{1}{2}\left(\mathcal{P}^{-1} \mathcal{F}\right)^{2}+\frac{1}{3}\left(\mathcal{P}^{-1} \mathcal{F}\right)^{3}+\cdots\right]
$$

In nonrelativistic theory, there are no anti-particles so that the loop diagrams are very limited compared to relativistic theory; the diagrams with anti-particle lines do not exist. The first term in (3.13) vanishes. (It is a field independent constant anyway.) The second term also vanishes because there is no way to draw the loop without an anti-particle line. The third and higher order terms contain the non-vanishing diagrams.

In the following sections, we concentrate on the two-nucleon sector, to which only the four-nucleon $(4 \mathrm{~N})$ operators contribute. It is easy to see that only the third term contains such contributions. (It also contains the contributions to the other sectors.) The other sectors get contributions from several terms in (3.13). To the three-nucleon sector, for example, both the third and the fourth terms contain the contributions.

In this way, we end up with the following reduced equation for the $4 \mathrm{~N}$ operators,

$$
\left.\frac{d \Gamma_{\Lambda}}{d t}\right|_{4 \mathrm{~N}}=-\left.\frac{i}{4} \operatorname{Tr} \tilde{\partial}_{t}\left[\left(\mathcal{P}^{-1} \mathcal{F}\right)^{2}\right]\right|_{4 \mathrm{~N}} .
$$

Although the above equation is exact, one needs an approximation to solve it. We consider a simple truncation of the space of operators. Namely, we consider only the operators of leading orders in derivative expansion. The approximation is based on our hope that, even 
though some operators get large anomalous dimensions, their "ordering" of importance would not change; the lower the canonical dimension is, the lower the scaling dimension would be. Of course some mixing of operators would occur and should be taken into account properly. There is no absolute justification for this hope. One should examine its validity by actually enlarging the space of operators and confirming the stability of the results against the enlargement.

An actual calculation goes as follows. We substitute the "ansatz" for the averaged action consisting of the operators up to a certain order into the above flow equation. The "ansatz" determines the explicit forms of $\mathcal{P}$ and $\mathcal{F}$. We then compare the coefficients of the operators on both sides. Higher oder operators which emerge in the RHS of (3.14) are disregarded ("projected out"). The LHS contains the derivatives of the couplings while the RHS does not, thus the comparison leads to a set of first-order differential equations for the couplings, the RG equations.

It is important to note that a WRG transformation generates all kinds of operators allowed by the symmetry of the theory including the so-called "redundant" operators, the operators which may be eliminated by the use of equations of motion. The "use of equations of motion" actually means a field redefinition, which eliminates the "redundant" term. In the previous paper 27], we showed that the field redefinition gives rise to a nontrivial Jacobian, and that "redundant" operators play an important role in a WRG analysis.

\section{RG ANALYSIS OF PIONLESS NEFT}

\section{A. pionless NEFT}

In this section, we consider the RG flow of the pionless NEFT, in which only nonrelativistic nucleons are treated as explicit degrees of freedom. Effects of anti-nucleons, pions, and other heavier mesons and baryons are integrated out and hidden in the values of the coupling constants. Thus the theory is expected to describe the interactions of nonrelativistic nucleons with external momenta sufficiently smaller than pion mass. Although our approach is general and not restricted to this theory, it serves as the simplest example which illustrates the essential points.

In a WRG analysis, symmetry is extremely important. We assume Galilean invariance, 
rotational symmetry, and invariance under charge conjugation, parity, and time-reversal. Furthermore, we assume exact isospin symmetry, and ignore electromagnetic and weak interactions. In the following, we will focus only on two channels, ${ }^{1} S_{0}$ and ${ }^{3} S_{1}-{ }^{3} D_{1}$, in the two-nucleon sector. The extension to higher partial waves is easy.

We study the RG flow for this theory by using Legendre flow equation. We consider the following "ansatz" for the averaged action, keeping only the operators with (canonical) dimension up to eight.

$$
\begin{aligned}
& \Gamma_{\Lambda}=\int d^{4} x\left[N^{\dagger}\left(i \partial_{t}+\frac{\nabla^{2}}{2 M}\right) N\right. \\
& \begin{cases}\left.-C_{0}^{(S)} \mathcal{O}_{0}^{(S)}+C_{2}^{(S)} \mathcal{O}_{2}^{(S)}+2 B^{(S)} \mathcal{O}_{2}^{(S B)}\right], & \left({ }^{1} S_{0} \text { channel }\right) \\
\left.-C_{0}^{(T)} \mathcal{O}_{0}^{(T)}+C_{2}^{(T)} \mathcal{O}_{2}^{(T)}+2 B^{(T)} \mathcal{O}_{2}^{(T B)}+C_{2}^{(S D)} \mathcal{O}_{2}^{(S D)}\right], & \left({ }^{3} S_{1}{ }^{3} D_{1} \text { channel }\right)\end{cases}
\end{aligned}
$$

where the operators in the ${ }^{1} S_{0}$ are given by

$$
\begin{aligned}
& \mathcal{O}_{0}^{(S)}=\left(N^{T} P_{a}^{\left(1 S_{0}\right)} N\right)^{\dagger}\left(N^{T} P_{a}^{\left({ }^{1} S_{0}\right)} N\right), \\
& \mathcal{O}_{2}^{(S)}=\left[\left(N^{T} P_{a}^{\left({ }^{1} S_{0}\right)} N\right)^{\dagger}\left(N^{T} P_{a}^{\left({ }^{1} S_{0}\right)} \overleftrightarrow{\nabla}^{2} N\right)+\text { h.c. }\right] \\
& \left.\mathcal{O}_{2}^{(S B)}=\left[\left\{N^{T} P_{a}^{\left(1 S_{0}\right)}\left(i \partial_{t}+\frac{\nabla^{2}}{2 M}\right) N\right\}^{\dagger}\left(N^{T} P_{a}^{(1} S_{0}\right) N\right)+h . c .\right] \text {, }
\end{aligned}
$$

and in the ${ }^{3} S_{1}-{ }^{3} D_{1}$ channel,

$$
\begin{aligned}
\mathcal{O}_{0}^{(T)} & =\left(N^{T} P_{i}^{\left({ }^{3} S_{1}\right)} N\right)^{\dagger}\left(N^{T} P_{i}^{\left({ }^{3} S_{1}\right)} N\right), \\
\mathcal{O}_{2}^{(T)} & =\left[\left(N^{T} P_{i}^{\left({ }^{3} S_{1}\right)} N\right)^{\dagger}\left(N^{T} P_{i}^{\left(3 S_{1}\right)} \overleftrightarrow{\nabla}^{2} N\right)+\text { h.c. }\right], \\
\mathcal{O}_{2}^{(S D)} & =\left[\left(N^{T} P_{i}^{\left({ }^{3} S_{1}\right)} N\right)^{\dagger}\left\{N^{T}\left(\overleftrightarrow{\nabla}_{i} \overleftrightarrow{\nabla}_{j}-\frac{1}{3} \delta_{i j} \overleftrightarrow{\nabla}^{2}\right) P_{j}^{\left({ }^{3} S_{1}\right)} N\right\}+\text { h.c. }\right], \\
\mathcal{O}_{2}^{(T B)} & =\left[\left\{N^{T} P_{i}^{\left({ }^{3} S_{1}\right)}\left(i \partial_{t}+\frac{\nabla^{2}}{2 M}\right) N\right\}^{\dagger}\left(N^{T} P_{i}^{\left({ }^{3} S_{1}\right)} N\right)+\text { h.c. }\right],
\end{aligned}
$$

where we have introduced the notation $\overleftrightarrow{\nabla}^{2} \equiv \overleftarrow{\nabla^{2}}+\vec{\nabla}^{2}-2 \overleftarrow{\nabla} \cdot \vec{\nabla}$ and the projection operators [20],

$$
P_{a}^{\left(1 S_{0}\right)} \equiv \frac{1}{\sqrt{8}} \sigma^{2} \tau^{2} \tau^{a}, \quad P_{k}^{\left({ }^{3} S_{1}\right)} \equiv \frac{1}{\sqrt{8}} \sigma^{2} \sigma^{k} \tau^{2},
$$

for the ${ }^{1} S_{0}$ channel and the ${ }^{3} S_{1}$ channel respectively. The nucleon field $N(x)$ with mass $M$ is an isospin doublet nonrelativistic two-component spinor. Pauli matrices $\sigma^{i}$ and $\tau^{a}$ act on 


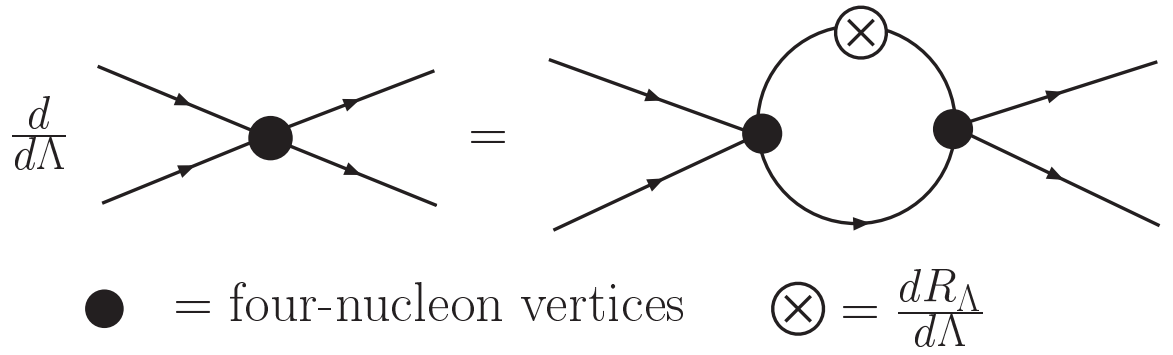

FIG. 1: The loop diagram which contributes to the Legendre flow equation for four-nucleon operators.

spin indices and isospin indices respectively. The two channels are completely decoupled, and thus we can consider each channel separately.

Note that we have not included the wave function renormalization because there is no such contribution due to the nonrelativistic feature. We neither include six-nucleon operators and higher because of the nucleon number conservation. The more-than-two-nucleon sectors are completely decoupled from the two-nucleon sector which we are interested in.

Note also that we have included redundant operators, $\mathcal{O}_{2}^{(S B)}$ and $\mathcal{O}_{2}^{(T B)}$, because they are necessary in a consistent WRG analysis.

Let us consider the simplest example of the loop calculation in order to explain how we maintain the Galilean invariance. The diagrams which contribute to the Legendre flow equation are of the type shown in FIG. 11, with various factors at the vertices. The propagator is IR cutoff by the function (3.5), so that we are interested in the following integral,

$$
I \equiv \int \frac{d^{4} k}{(2 \pi)^{4}} \frac{i}{P^{0} / 2+k^{0}-\mathcal{R}\left((\boldsymbol{P} / 2+\boldsymbol{k})^{2}\right)+i \epsilon} \cdot \frac{i}{P^{0} / 2-k^{0}-\mathcal{R}\left((\boldsymbol{P} / 2-\boldsymbol{k})^{2}\right)+i \epsilon},
$$

where we have introduced

$$
\mathcal{R}\left(\boldsymbol{k}^{2}\right)=\frac{\boldsymbol{k}^{2}}{2 M}-R_{\Lambda}^{(1)}\left(\boldsymbol{k}^{2}\right)
$$

and $\left(P^{0}, \boldsymbol{P}\right)$ is the momentum flowing in (and out of) the diagram. Note that we assign the loop momentum $\left(k^{0}, \boldsymbol{k}\right)$ so that $I$ is invariant under $\boldsymbol{P} \rightarrow-\boldsymbol{P}$. Note also that there may be additional momentum factors from the vertices but they do not affect the following argument. After integrating $k^{0}$, we have

$$
I=\int \frac{d^{3} k}{(2 \pi)^{3}} \frac{i}{P^{0}-\mathcal{R}\left((\boldsymbol{P} / 2-\boldsymbol{k})^{2}\right)-\mathcal{R}\left((\boldsymbol{P} / 2+\boldsymbol{k})^{2}\right)+i \epsilon} .
$$


It is important to note that, if the IR cutoff were absent, the propagator would be

$$
\frac{i}{P^{0}-\frac{\boldsymbol{P}^{2}}{4 M}-\frac{\boldsymbol{k}^{2}}{M}+i \epsilon},
$$

where the combination $\boldsymbol{P}^{2} / 4 M$ describes the center-of-mass kinetic energy of the two nucleons while $\boldsymbol{k}^{2} / M$ represents the kinetic energy of the relative motion. It is now clear that the only way to maintain the Galilean invariance is to cutoff the relative momentum. It is therefore physically legitimate to replace (4.7) with

$$
\int \frac{d^{3} k}{(2 \pi)^{3}} \frac{i}{P^{0}-\frac{\boldsymbol{P}^{2}}{4 M}-\frac{\boldsymbol{k}^{2}}{M}+2 R_{\Lambda}^{(1)}\left(\boldsymbol{k}^{2}\right)+i \epsilon} .
$$

The integral may be evaluated with an arbitrary parameter $n$. A useful formula for a typical loop integral is given in Appendix A1 In the following, we consider only the sharp cutoff limit $n \rightarrow \infty$, for which the RG equation becomes the simplest. We also look at the dependence of the fixed points and the anomalous dimensions against the variation of $n$. They are shown in Appendix A2.

\section{B. RG flows and fixed points}

It is useful to define the dimensionless variables as follows and write the RG equation in terms of these variables. In the ${ }^{1} S_{0}$ channel, we introduce ${ }^{3}$,

$$
x \equiv \frac{M \Lambda}{2 \pi^{2}} C_{0}^{(S)}, \quad y \equiv \frac{M \Lambda^{3}}{2 \pi^{2}} 4 C_{2}^{(S)}, \quad z \equiv \frac{\Lambda^{3}}{2 \pi^{2}} B^{(S)},
$$

in terms of which the RG equations are given by

$$
\begin{aligned}
& \frac{d x}{d t}=-x-\left[x^{2}+2 x y+y^{2}+2 x z+2 y z+z^{2}\right], \\
& \frac{d y}{d t}=-3 y-\left[\frac{1}{2} x^{2}+2 x y+\frac{3}{2} y^{2}+y z-\frac{1}{2} z^{2}\right], \\
& \frac{d z}{d t}=-3 z+\left[\frac{1}{2} x^{2}+x y+\frac{1}{2} y^{2}-x z-y z-\frac{3}{2} z^{2}\right] .
\end{aligned}
$$

\footnotetext{
${ }^{3}$ The factor $M$ comes from the scale transformation property in nonrelativistic theory, $t^{\prime}=\lambda^{2} t, \boldsymbol{x}^{\prime}=$ $\lambda \boldsymbol{x}$, where $\lambda$ is the scale factor. Under this transformation, the nucleon field transforms as $N(t, \boldsymbol{x})=$ $\lambda^{\frac{3}{2}} N^{\prime}\left(t^{\prime}, \boldsymbol{x}^{\prime}\right)$, and thus we have $\mathcal{O}_{0}^{(S)}=\lambda^{6} \mathcal{O}^{\prime}{ }_{0}^{(S)}$ for example. The $d^{4} x$ gives the factor $\lambda^{-5}$, and the additional factor $\lambda^{-1}$ is supplied by the $\Lambda$-dependence of the coupling $C_{0}^{(S)} \propto \Lambda^{-1}$ to cancel the $\lambda^{6}$. To have the right mass dimension, $C_{0}^{(S)}$ has the $1 / M$ dependence. Similar consideration leads to the correct $\Lambda$ and $M$ dependence of other couplings.
} 
Note that the RG equations are quadratic in four-nucleon couplings due to the nonexistence of anti-nucleons in our nonrelativistic formulation, up to the first terms, which come from the canonical scaling.

Similarly in the ${ }^{3} S_{1}-^{3} D_{1}$ channel, we introduce

$$
x^{\prime} \equiv \frac{M \Lambda}{2 \pi^{2}} C_{0}^{(T)}, \quad y^{\prime} \equiv \frac{M \Lambda^{3}}{2 \pi^{2}} 4 C_{2}^{(T)}, \quad z^{\prime} \equiv \frac{\Lambda^{3}}{2 \pi^{2}} B^{(T)}, \quad w^{\prime} \equiv \frac{M \Lambda^{3}}{2 \pi^{2}} \frac{4}{3} C_{2}^{(S D)},
$$

and we have the following RG equations,

$$
\begin{aligned}
& \frac{d x^{\prime}}{d t}=-x^{\prime}-\left[x^{\prime 2}+2 x^{\prime} y^{\prime}+y^{\prime 2}+2 x^{\prime} z^{\prime}+2 y^{\prime} z^{\prime}+z^{\prime 2}+2 w^{\prime 2}\right], \\
& \frac{d y^{\prime}}{d t}=-3 y^{\prime}-\left[\frac{1}{2} x^{\prime 2}+2 x^{\prime} y^{\prime}+\frac{3}{2} y^{\prime 2}+y^{\prime} z^{\prime}-\frac{1}{2} z^{\prime 2}+w^{\prime 2}\right], \\
& \frac{d z^{\prime}}{d t}=-3 z^{\prime}+\left[\frac{1}{2} x^{\prime 2}+x^{\prime} y^{\prime}+\frac{1}{2} y^{\prime 2}-x^{\prime} z^{\prime}-y^{\prime} z^{\prime}-\frac{3}{2} z^{\prime 2}+w^{\prime 2}\right], \\
& \frac{d w^{\prime}}{d t}=-3 w^{\prime}-\left[x^{\prime} w^{\prime}+y^{\prime} w^{\prime}+z^{\prime} w^{\prime}\right] .
\end{aligned}
$$

Note that the RG equations (4.13) for the ${ }^{3} S_{1}{ }^{-3} D_{1}$ channel are identical to the RG equations (4.11) for the ${ }^{1} S_{0}$ channel if $w^{\prime}$ is set equal to zero. (This is actually a solution.)

We can now draw the RG flow diagram. FIG. 2 shows the RG flow for the ${ }^{1} S_{0}$ channel and FIG. [3 for the ${ }^{3} S_{1}{ }^{-3} D_{1}$ channel up to operators with dimension eight. Both flows are projected on to the two-dimensional plane spanned by the lowest order coupling constants. As expected from the RG equations (4.11) and (4.13), the RG flows for the ${ }^{1} S_{0}$ channel and for the ${ }^{3} S_{1}{ }^{-3} D_{1}$ channel have similar structure. First of all, there are two fixed points in both RG flows; the trivial IR fixed point and the non-trivial UV fixed point. Secondly, both RG flows have two phases; one in which all points approaches the trivial fixed point in the limit of vanishing cutoff, and the other one in which all points approaches infinity at certain finite cutoff scale. The nontrivial fixed points are on the phase boundaries.

Location of the fixed points are given by solutions of the equations, $d x / d t=d y / d t=$ $d z / d t=0$, for the ${ }^{1} S_{0}$ channel, and $d x^{\prime} / d t=d y^{\prime} / d t=d z^{\prime} / d t=d w^{\prime} / d t=0$ for the ${ }^{3} S_{1}-{ }^{3} D_{1}$ channel. As explained in Appendix A2, we find four fixed points in each channel for an arbitrary value of the parameter $n$. One of them is extremely unstable against the variation of $n$, and may be considered as "spurious", i.e., it is an artifact of our truncation. The rest are stable and take the following values in the $n \rightarrow \infty$ limit, 


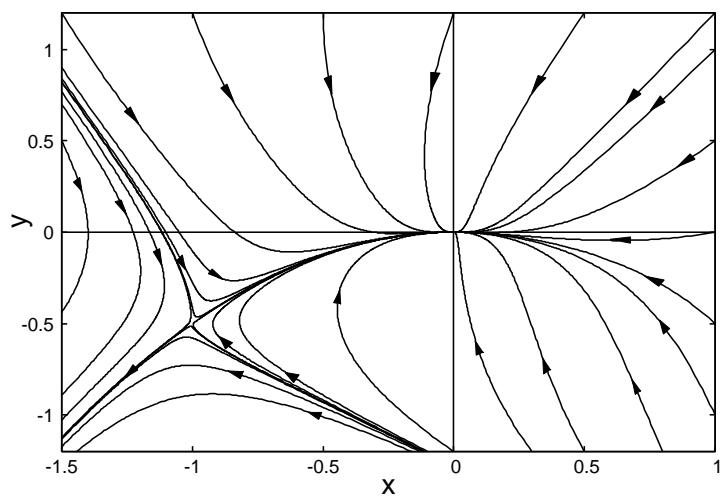

FIG. 2: The RG flow for the ${ }^{1} S_{0}$ channel projected onto the $C_{0}^{(S)}-C_{2}^{(S)}$ plane. Flow lines start with the arbitrarily chosen value $z=0$ at the edges of the graph.

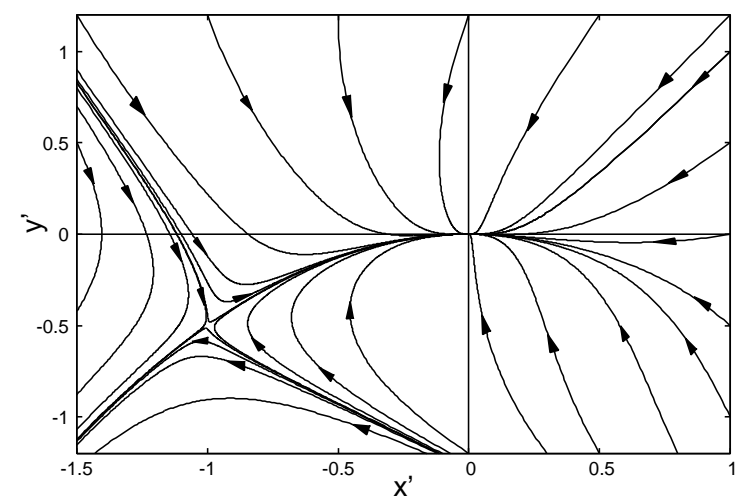

FIG. 3: The RG flow for the ${ }^{3} S_{1}{ }^{3} D_{1}$ channel projected onto the $C_{0}^{(T)}-C_{2}^{(T)}$ plane. Flow lines start with the arbitrarily chosen values $z^{\prime}=0$ and $w^{\prime}=0.1$ at the edges of the graph.

${ }^{1} S_{0}$ channel:

$$
\left(x^{\star}, y^{\star}, z^{\star}\right)=(0,0,0), \quad\left(-1,-\frac{1}{2}, \frac{1}{2}\right), \quad\left(-9, \frac{15}{2},-\frac{3}{2}\right)
$$

$\underline{{ }^{3} S_{1}{ }^{3} D_{1} \text { channel: }}$

$$
\left(x^{\prime \star}, y^{\prime \star}, z^{\prime \star}, w^{\prime \star}\right)=(0,0,0,0), \quad\left(-1,-\frac{1}{2}, \frac{1}{2}, 0\right), \quad\left(-9, \frac{15}{2},-\frac{3}{2}, 0\right) .
$$

We also disregard the third fixed points in both channels, because they are irrelevant to the discussion of the power counting. The eigenoperators at these fixed points are complex and have complex eigenvalues (scaling dimensions). It means that the operators do not have the definite scaling property there.

We obtain the scattering amplitudes explicitly in both channels in Appendix $\mathbb{B}$ as we did in the previous paper[27], and find that the RG equations, which look much more complicated than (4.11) and (4.13), have only two fixed points in each channel and they exactly agree with the first two fixed points obtained here ${ }^{4}$.

\footnotetext{
${ }^{4}$ Actually, the RG equation obtained in the previous paper [27] is those for the ${ }^{1} S_{0}$ channel.
} 


\section{C. scaling dimensions}

We now study the behavior of the coupling constants around each fixed point by considering the linearized RG equations.

Let us first consider the trivial fixed points. In the ${ }^{1} S_{0}$ channel, the trivial fixed point is at $\left(x^{\star}, y^{\star}, z^{\star}\right)=(0,0,0)$. We consider a small deviation $(\delta x, \delta y, \delta z)$ around it and linearize the RG equations (4.11),

$$
\frac{d}{d t}\left(\begin{array}{c}
\delta x \\
\delta y \\
\delta z
\end{array}\right)=\left(\begin{array}{ccc}
-1 & 0 & 0 \\
0 & -3 & 0 \\
0 & 0 & -3
\end{array}\right)\left(\begin{array}{c}
\delta x \\
\delta y \\
\delta z
\end{array}\right)
$$

The eigenvalues and corresponding eigenvectors are trivially obtained,

$$
\nu_{1}=-1: u_{1}=\left(\begin{array}{l}
1 \\
0 \\
0
\end{array}\right), \quad \nu_{2}=-3: \quad u_{2}=\left(\begin{array}{l}
0 \\
1 \\
0
\end{array}\right), \quad \nu_{3}=-3: u_{3}=\left(\begin{array}{l}
0 \\
0 \\
1
\end{array}\right) \text {. }
$$

Namely, the couplings $C_{0}^{(S)}, C_{2}^{(S)}$, and $B^{(S)}$ have the scaling dimension $-1,-3$, and -3 respectively. This is what one naively expects from their canonical scaling properties.

Similarly in the ${ }^{3} S_{1}{ }^{3} D_{1}$ channel, we consider a deviation $\left(\delta x^{\prime}, \delta y^{\prime}, \delta z^{\prime}, \delta w^{\prime}\right)$ around the trivial fixed point $\left(x^{\prime \star}, y^{\prime \star}, z^{\prime \star}, w^{\prime \star}\right)=(0,0,0,0)$, and find the following linearized RG equations from Eq. (4.13),

$$
\frac{d}{d t}\left(\begin{array}{c}
\delta x^{\prime} \\
\delta y^{\prime} \\
\delta z^{\prime} \\
\delta w^{\prime}
\end{array}\right)=\left(\begin{array}{cccc}
-1 & 0 & 0 & 0 \\
0 & -3 & 0 & 0 \\
0 & 0 & -3 & 0 \\
0 & 0 & 0 & -3
\end{array}\right)\left(\begin{array}{c}
\delta x^{\prime} \\
\delta y^{\prime} \\
\delta z^{\prime} \\
\delta w^{\prime}
\end{array}\right)
$$


with the eigenvalues and corresponding eigenvectors,

$$
\begin{aligned}
& \nu_{1}=-1: u_{1}=\left(\begin{array}{l}
1 \\
0 \\
0 \\
0
\end{array}\right), \quad \nu_{2}=-3: u_{2}=\left(\begin{array}{l}
0 \\
1 \\
0 \\
0
\end{array}\right), \nu_{3}=-3: u_{3}=\left(\begin{array}{l}
0 \\
0 \\
1 \\
0
\end{array}\right) \\
& \nu_{4}=-3: u_{4}=\left(\begin{array}{l}
0 \\
0 \\
0 \\
1
\end{array}\right)
\end{aligned}
$$

The coupling constants $C_{0}^{(T)}, C_{2}^{(T)}, B^{(T)}$, and $C_{2}^{(S D)}$ have the scaling dimension $-1,-3,-3$, and -3 respectively.

Note that all of the scaling dimensions are negative at the trivial fixed point and thus the corresponding operators are irrelevant, i.e., they should be treated perturbatively.

At the nontrivial fixed point, on the other hand, something much more interesting happens. In the ${ }^{1} S_{0}$ channel, the deviations from the nontrivial fixed point $\left(x^{\star}, y^{\star}, z^{\star}\right)=$ $(-1,-1 / 2,1 / 2)$ satisfy the following linearized $\mathrm{RG}$ equations,

$$
\frac{d}{d t}\left(\begin{array}{c}
\delta x \\
\delta y \\
\delta z
\end{array}\right)=\left(\begin{array}{ccc}
1 & 2 & 2 \\
2 & 0 & 1 \\
-2 & -2 & -3
\end{array}\right)\left(\begin{array}{c}
\delta x \\
\delta y \\
\delta z
\end{array}\right)
$$

with the eigenvalues and corresponding eigenvectors,

$$
\nu_{1}=+1: u_{1}=\left(\begin{array}{c}
1 \\
1 \\
-1
\end{array}\right), \quad \nu_{2}=-1: u_{2}=\left(\begin{array}{c}
0 \\
-1 \\
1
\end{array}\right), \quad \nu_{3}=-2: u_{3}=\left(\begin{array}{c}
2 \\
-1 \\
-2
\end{array}\right) \text {. }
$$

Note that the scaling dimensions change drastically. Note also that the eigenoperators are not monomials but linear combinations of the operators. This is a typical feature of the momentum cutoff regularization.

Similar results are obtained in the ${ }^{3} S_{1}{ }^{3} D_{1}$ channel, where the linearized equations around 
the nontrivial fixed point $\left(x^{\prime \star}, y^{\prime \star}, z^{\prime \star}, w^{\prime \star}\right)=(-1,-1 / 2,1 / 2,0)$ become

$$
\frac{d}{d t}\left(\begin{array}{c}
\delta x^{\prime} \\
\delta y^{\prime} \\
\delta z^{\prime} \\
\delta w^{\prime}
\end{array}\right)=\left(\begin{array}{cccc}
1 & 2 & 2 & 0 \\
2 & 0 & 1 & 0 \\
-2 & -2 & -3 & 0 \\
0 & 0 & 0 & -2
\end{array}\right)\left(\begin{array}{c}
\delta x^{\prime} \\
\delta y^{\prime} \\
\delta z^{\prime} \\
\delta w^{\prime}
\end{array}\right),
$$

giving the following eigenvalues and corresponding eigenvectors,

$$
\begin{aligned}
& \nu_{1}=+1: u_{1}=\left(\begin{array}{c}
1 \\
1 \\
-1 \\
0
\end{array}\right), \quad \nu_{2}=-1: u_{2}=\left(\begin{array}{c}
0 \\
-1 \\
1 \\
0
\end{array}\right), \nu_{3}=-2: u_{3}=\left(\begin{array}{c}
2 \\
-1 \\
-2 \\
0
\end{array}\right) \\
& \nu_{4}=-2: u_{4}=\left(\begin{array}{l}
0 \\
0 \\
0 \\
1
\end{array}\right)
\end{aligned}
$$

Note that the structure is essentially the same as that in the ${ }^{1} S_{0}$ channel, with $\delta w^{\prime}$ decoupled.

The most important result at the nontrivial fixed point is that there is a relevant coupling in each channel. The coupling $u_{1}$ has dimension +1 , and according to the argument given in Sec. III the corresponding operator should be treated nonperturbatively, while the others perturbatively. The operators should be ordered according to the scaling dimensions. The less the dimension of a coupling constant is, the less the importance of the corresponding operator is.

As we have done in the previous paper [27], RG equations may be obtained by directly calculating the amplitudes and requiring them to be independent of the "floating" scale $\Lambda$. See Appendix $\mathrm{B}$ for the results. The resulting $\mathrm{RG}$ equations are much more complicated. It is interesting, however, the location of the fixed points and the scaling dimensions are precisely the same as those obtained here with the Legendre flow equation. The eigenvectors are a bit different, though.

\section{D. physical meaning of the RG phases}

Let us consider the real world and examine the physical meaning of what we have found in the two-nucleon system. 
As mentioned in Introduction, the two-nucleon system is considered as "fine-tuned," in the sense that the scattering length is unnaturally large. It suggests that the actual system is near the nontrivial fixed point in each channel. The closer to the fixed point it is, the longer the scattering length is.

What then makes the physical difference between the ${ }^{1} S_{0}$ and the ${ }^{3} S_{1}-{ }^{3} D_{1}$ channels, both of which are close to the nontrivial fixed points? The answer to this question is provided by the flow diagram; the nontrivial fixed point is on the boundary between two phases. In one of them, the flow goes toward the trivial fixed point in the IR. We therefore call this the weak coupling phase. In the other, the flow goes toward stronger couplings, thus we call it the strong coupling phase. It is natural to think that the real two-nucleon system in the ${ }^{1} S_{0}$ channel, in which there is no bound state, is in the weak coupling phase, while that in the ${ }^{3} S_{1}{ }^{3} D_{1}$ channel, in which there is a shallow bound state, deuteron, is in the strong coupling phase.

In order to make the physical picture more transparent, it is useful to examine the fournucleon (two-nucleon scattering) amplitudes. As shown in Ref. 27] and in Appendix B, the four-nucleon amplitudes may be obtained explicitly in the derivative expansion. By substituting the solution of the linearized RG equation in the ${ }^{1} S_{0}$ channel obtained from the direct method,

$$
\left(\begin{array}{c}
\delta x \\
\delta y \\
\delta z
\end{array}\right)=a\left(\begin{array}{c}
2 \\
1 \\
-4
\end{array}\right)\left(\frac{\Lambda}{\Lambda_{0}}\right)^{2}+b\left(\begin{array}{c}
0 \\
-1 \\
1
\end{array}\right)\left(\frac{\Lambda}{\Lambda_{0}}\right)+c\left(\begin{array}{c}
1 \\
1 \\
-1
\end{array}\right)\left(\frac{\Lambda_{0}}{\Lambda}\right)
$$

where $a, b$, and $c$ are small dimensionless constants, into the amplitude in the center-of-mass frame, we obtained the renormalized (off-shell) amplitude near the nontrivial fixed point [27],

$$
\left.\mathcal{A}^{-1}\left(p^{0}, \boldsymbol{p}^{\prime 2}, \boldsymbol{p}^{2}\right)\right|_{*}=-\frac{M}{4 \pi}\left[\frac{2 c}{\pi} \Lambda_{0}-\frac{4 b}{\pi}\left(\frac{M p^{0}}{\Lambda_{0}}\right)-i \sqrt{M p^{0}}\right]+\cdots
$$

where ellipsis denotes higher orders in $1 / \Lambda_{0}$.

By comparing it with the effective range expansion,

$$
\left.\mathcal{A}^{-1}\right|_{\text {on-shell }}=-\frac{M}{4 \pi}\left[-\frac{1}{\alpha}+\frac{1}{2} r p^{2}-i p\right]+\cdots,
$$

on the mass shell $p=\sqrt{M p^{0}}=|\boldsymbol{p}|=\left|\boldsymbol{p}^{\prime}\right|$, one sees the scattering length $\alpha$ and the effective 
range $r$ are given by

$$
\alpha=-\frac{\pi}{2 c \Lambda_{0}}, \quad r=-\frac{8 b}{\pi \Lambda_{0}} .
$$

That is, the inverse of the scattering length is identified as the relevant coupling. The finetuning $|\alpha| \gg \Lambda_{0}^{-1}$ indeed corresponds to $|c| \ll 1$. It is also important that the sign of the coupling $c$ distinguishes the phases, namely, the inverse of the scattering length is the order parameter. It vanishes on the boundary, the critical surface. Because the effective range is of natural size, $r \sim \Lambda_{0}^{-1}$, it is more accurate to say that the real two-nucleon system is close to the critical surface.

The location of the pole which is found within the range of EFT is given by

$$
p_{0} \simeq-\frac{\Lambda_{0}^{2}}{M}\left(\frac{2 c}{\pi}\right)^{2}\left[1+\frac{16 b c}{\pi^{2}}\right] .
$$

A similar expression is obtained for the pole in the ${ }^{3} S_{1}-{ }^{3} D_{1}$ channel. The pole on the physical Riemann sheet that corresponds to a bound state occurs only when $c<0$, i.e., in the strong coupling phase, while there is no restriction on $b$.

\section{COMMENTS ON OTHER POWER COUNTING SCHEMES}

As we emphasized in Sec. III, our way of determining the power counting on the basis of the scaling dimensions is general and nonperturbative. It does not depend on any specific regularization/renormalization scheme, nor on which fixed point we are looking at.

In this section, we would like to comment on other works from our point of view and make the connection between their power counting and ours. It leads us to a deeper understanding of the power counting issues.

First of all, we would like to mention the work done by Birse, McGovern, and Richardson [26], which is very close to ours in philosophy. They consider the most general potential and the Wilsonian RG equation satisfied by it. The "fixed point" potential depends only on the energy,

$$
V_{0}(p, \Lambda)=-\frac{2 \pi^{2}}{M}\left[\Lambda-\frac{p}{2} \ln \frac{\Lambda+p}{\Lambda-p}\right]^{-1},
$$

where $p=\sqrt{M E}$, with the $E$ being the center-of-mass energy. They then consider the perturbation around the "fixed point" potential, and find the eigenvalues of the linearized RG equations. The first few eigenvalues coincide with ours. They use the information to 
determine the power counting. It is important to note that the "energy-dependent perturbations" have the spectrum $\nu=-1,1,3, \cdots$, while the "momentum-dependent perturbations" have $\nu=2,4,5, \cdots$. (Note that their $\nu$ 's have opposite signs to the scaling dimensions used in this paper.)

To compare their results with ours, it is useful to translate our operator formulation to the potential one. Our operators may be equivalent to the potential,

$$
V\left(p^{0}, \boldsymbol{p}, \boldsymbol{p}^{\prime}\right)=C_{0}+4 C_{2}\left(\boldsymbol{p}^{2}+\boldsymbol{p}^{\prime 2}\right)-2 B\left(p^{0}-\frac{\boldsymbol{p}^{2}+\boldsymbol{p}^{2}}{2 M}\right)+\cdots,
$$

where we suppress the superscripts which denote the channel. If we substitute the fixed point values of the couplings, we have

$$
V^{\star}\left(p^{0}, \boldsymbol{p}, \boldsymbol{p}^{\prime}\right)=-\frac{2 \pi^{2}}{M \Lambda}\left[1+\frac{M p^{0}}{\Lambda^{2}}\right]+\cdots,
$$

which is exactly the expansion of the potential (5.1), with $p^{0}$ replaced by $E$. Note that the momentum dependence cancels at the fixed point. This may be an evidence that both approaches are essentially the same. Actually, the "perturbations" with the scaling dimensions $\nu_{1}=1$ and $\nu_{2}=-1$ corresponds to the potentials that depends only on $p^{0}$, while that with $\nu_{3}=-2$ to a momentum-dependent one, in agreement with their findings ${ }^{5}$.

For this simplest pionless NEFT in the two-nucleon sector, the method based on the potential has an advantage. On the other hand, because our approach is based on the general principle of fully-fledged quantum field theory, we could treat more complicated systems in a unified and systematic way. For example, we could treat three-nucleon problems without worrying about the off-shell ambiguities, and fully relativistic systems for which the potential picture is not valid. The important point is that our work gives a field-theoretical foundation for their approach and has opened a window to wider applications.

In the following, we will make comments on other power counting schemes on the basis of what we have found.

In Weinberg's scheme [5, 6], one assumes the NDA power counting in making the "effective potential," then treats it nonperturbatively. As we have explained, however, the NDA is the power counting near the trivial fixed point, where all the operators are irrelevant and should

\footnotetext{
${ }^{5}$ A careful study reveals that the eigenvector for $\nu_{3}=-2$ does not agree with theirs, though those for $\nu_{1}=1$ and $\nu_{2}=-1$ do. The reason seems that the reaction matrix they consider does not have a direct connection to the scattering amplitude which we work with off the mass shell.
} 
be treated perturbatively. The so-called infrared enhancement due to two-nucleon reducible diagrams somehow remedies the above-mentioned mismatch in the treatment; it makes the "effective potential" more relevant. The way is not systematic however, and in some cases inconsistent [16].

The scheme due to Kaplan, Savage, and Wise 18, 19] is important because they emphasized one of the most important aspects of the two-nucleon system; it is close to the nontrivial fixed point! (The existence of the nontrivial fixed point itself had been noticed by Weinberg[6].) It is however very subtle why the KSW scheme succeeds in some channels, and fails in others. To understand the points, we have to examine it very carefully.

It is difficult to define the KSW power counting without mentioning the Power Divergence Subtraction (PDS) scheme ${ }^{6}$. In PDS, the poles at $D=3$ as well as $D=4$ are subtracted. In the pionless NEFT, there is no pole at $D=4$, so that one subtracts as if one were in $D=3$ dimensions. This is the heart of the PDS scheme; it changes the (canonical) dimensions of the operators! For example, the operator $\mathcal{O}_{0}^{(S)}$ has the canonical dimension 6 in $D=4$ dimensions, but would have 4 in $D=3$ dimensions. In PDS, it is treated as an operator of dimension 4. This shift of the dimension by two coincides with the correct anomalous dimension of the operator, as we found in the previous section. (Remember that the leading contact operators have anomalous dimension 2, and according to Birse et al., all the "energy-dependent perturbations" seem to have the same anomalous dimensions.) We believe that it is the true reason why the KSW/PDS scheme works near the nontrivial fixed point $^{7}$.

Actually, this "shift by two" applies to all the four-nucleon operators in the KSW/PDS scheme $^{8}$. To see this, consider the KSW/PDS RG equation for the dimensionless couplings,

$$
\mu \frac{d \gamma_{2 n}}{d \mu}=(2 n+1) \gamma_{2 n}+\sum_{m=0}^{n} \gamma_{2 m} \gamma_{2(n-m)},
$$

where $\gamma_{2 n} \equiv\left(M \mu^{2 n+1} / 4 \pi\right) C_{2 n}$, with $C_{2 n}(\mu)$ being given in Eq. (2.10) in Ref. [19]. Near the

\footnotetext{
${ }^{6}$ We do not claim that PDS is indispensable for the KSW power counting, but that the PDS scheme provides the most unambiguous definition of the KSW power counting.

${ }^{7}$ From this point of view, it is legitimate to think that the KSW/PDS scheme should be applied only to the case near the nontrivial fixed point, though the RG equations (5.4) know about the trivial fixed point too.

8 The redundant operators scales differently than the others, but they are not necessary with dimensional regularization.
} 
nontrivial fixed point $\left(\gamma_{0}^{\star}, \gamma_{2}^{\star}, \gamma_{4}^{\star}, \cdots\right)=(-1,0,0, \cdots)$, the RHS of (5.4) becomes

$$
(2 n+1) \gamma_{2 n}+2 \gamma_{0} \gamma_{2 n}+\cdots \simeq(2 n-1) \gamma_{2 n},
$$

where the ellipsis stands for terms quadratic in $\gamma^{\prime}$ s and independent of $\gamma_{0}$, which do not contribute to the anomalous dimensions. This explicitly shows the shift from $(2 n+1)$ to $(2 n-1)^{9}$.

As far as all the anomalous dimensions are two, the KSW/PDS scheme would work perfectly. The problem in the pionful NEFT seems that the singular interactions due to pion exchange in the ${ }^{3} S_{1} \_{ }^{3} D_{1}$ channel would modify the anomalous dimensions of the contact operators strongly, making the "shift by two" rule invalid. Actually, Birse 22] found that the anomalous dimensions shift additionally by minus one half at the nontrivial fixed point in the presence of the tensor force due to one-pion exchange. The investigation in this direction is currently in progress [31].

Several authors considered power countings which are compatible with the KSW power counting without using PDS so that one might think that the KSW scheme may be defined independent of PDS. In fact, it appears possible to do so if one assumes the scaling of the coupling constants which is a consequence of the PDS regularization/renormalization. In

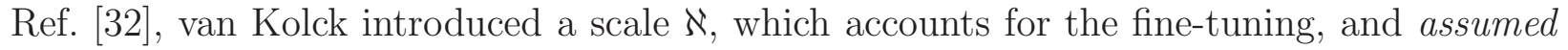
the scaling of the coupling constants without specifying the regularization/renormalization. The scale $\aleph$ seems to correspond to our $c \Lambda_{0}$ with $c \ll 1$ introduced in Eq. (4.24). It is important to note that $\aleph$ is a physical scale so that there is no notion of RG with respect to $\aleph$ in his approach. If one nevertheless identifies $\aleph$ with the scale $\mu$ in the KSW scheme, the assumed scaling of the couplings is just the one in the KSW/PDS scheme. On the other hand, using momentum space subtraction renormalization, Mehen and Stewart [33, 34] imposed the renormalization condition (Eq. (6) in Ref. [33])

$$
\left.i A^{(m-1)}\right|_{p=i \mu_{R}, m_{\pi}=0}=-i C_{2 m}\left(\mu_{R}\right)\left(i \mu_{R}\right)^{2 m},
$$

\footnotetext{
9 There is a subtle point about the scaling of the coupling constants. If one solves the RG equations near the fixed point, the coupling constant scales $C_{2 n} \sim 4 \pi / M \Lambda_{0}^{2 n-1} \mu^{2}$, but it is different from the KSW scaling $C_{2 n} \sim 4 \pi / M \Lambda_{0}^{n} \mu^{n+1}$. It is due to the assumption that the effective range is of natural size, $r \sim 1 / \Lambda_{0}$. Namely, the KSW scaling is not that for a point close to the fixed point, but for a point close to the critical surface.
} 
on the (part of the) amplitude that scales as $Q^{m-1}$, where $Q$ is a typical momentum scale. (This power counting for the amplitudes is also assumed in accordance with the KSW/PDS scheme.) Putting $\mu_{R} \sim Q$, it requires the coupling constant $C_{2 m}\left(\mu_{R}\right)$ to scale as $\sim Q^{m-1}\left(\mu_{R}\right)^{-2 m} \sim Q^{-(m+1)}$, which is essentially equivalent to the KSW/PDS power counting. It is still unclear to us why the renormalization condition (5.6) reflects the situation that we are close to the nontrivial fixed point.

Let us finally comment on the confusion in the literature over the scale $\mu$ introduced in dimensional regularization. It is important to note that the scale $\mu$ does not have intrinsic meaning of "typical scale." It is completely arbitrary and physics is independent of it. In the usual applications of dimensional regularization to relativistic field theory, $\mu$ appears in logarithms as $\ln \left(Q^{2} / \mu^{2}\right)$, where $Q$ is the typical scale of the process. It is this dependence that makes it useful to think $\mu \sim Q$ in order to suppress the "large logarithms." In our application to NEFT, however, there is no logarithm, thus no reason to think $\mu \sim Q$.

\section{CONCLUSION}

In this paper we studied the Wilsonian RG flow of pionless NEFT using the Legendre flow equation in order to determine the correct power counting. We emphasized that the determination of power counting should be based on the scaling dimensions, because power counting is in essence the order of magnitude estimate based on dimensional analysis, and the scaling dimensions are quantum dimensions of the operators.

The RG flow has a nontrivial fixed point as well as a trivial one in each of the ${ }^{1} S_{0}$ and ${ }^{3} S_{1}{ }^{3} D_{1}$ channels. Near the nontrivial fixed point, we found a relevant operator, the coupling constant of which is proportional to the inverse of the scattering length. The nontrivial fixed point is on the boundary of two phases, the weak coupling and the strong coupling phases. The inverse of the scattering length is regarded as the order parameter, which takes zero at the boundary. The real two-nucleon systems are considered to be close to the nontrivial fixed point.

The difference between the ${ }^{1} S_{0}$ and ${ }^{3} S_{1}{ }^{3} D_{1}$ channels comes from the difference of the phases they are in. In the strong coupling phase, the scattering length is positive and the four-nucleon amplitude has a physical pole which represent a bound state, the deuteron in

the ${ }^{3} S_{1}{ }^{3} D_{1}$ channel, while in the weak coupling phase, the scattering length is negative and 
there is no bound state; this phase describes the ${ }^{1} S_{0}$ channel. We believe that this way of viewing the difference between the two channels is new, and we expect that it would give some further insight into the character of nuclear force.

The relation to the work by Birse et al. was clarified. The use of fully developed EFT framework was emphasized. We also made comments on other power counting schemes and tried to understand why they succeed in some cases and fail in others from our point of view. In particular, we claimed that the true reason why the KSW/PDS scheme works is identified as the "shift by two," namely, PDS happens to capture the essential feature that the anomalous dimensions of the contact two-nucleon interactions are two.

It was essential to include the so-called redundant operators to consistently perform the Wilsonian RG analysis 27] in a fully field theoretical manner. They are also necessary to renormalize the off-shell amplitudes. The inclusion of them seems essential to consider the tree-nucleon systems.

The RG equations derived from the Legendre flow equation are much simpler than those from the amplitude, while the important physical information (the existence and the location of fixed points, the global structure of the flows, the scaling dimensions, etc.) is the same. The use of the Legendre flow equation would help us to tackle more difficult problems, where the direct method seems infeasible.

It is very important to apply the present method based on the Wilsonian RG to determine the power counting of the pionful NEFT, where the power counting issues are most controversial. The singular tensor force would change the anomalous dimension of the lowest-order contact operator drastically in the ${ }^{3} S_{1}{ }^{3} D_{1}$ channel, invalidating the "shift by two" rule. One might imagine that, at a high energy scale, there is actually no difference of the values of couplings between the ${ }^{1} S_{0}$ and ${ }^{3} S_{1}{ }^{3} D_{1}$ channels, but the pion interactions make them so different. This scenario appears attractive from the approximate spin-flavor symmetry point of view [35, 36, 37]. Note that the nontrivial fixed point is spin-flavor symmetric.

One of the interesting results in this paper is the explicit relation between the Wilsonian $\mathrm{RG}$ analysis and the existence of the bound state in the strong coupling phase. It would open a new possibility of studying bound states using the Wilsonian RG. On the other hand, it would become important when we study the tree-nucleon systems without introducing a "dibaryon" field [38, 39, 40]. Note that most of the existing literature on the NEFT study of three-nucleon systems is based on the "dibaryon" formalism. Because our formulation is 
fully field theoretical, it is free from the off-shell ambiguities which the approaches based on potentials suffer from.

\section{Acknowledgments}

We would like to thank Y. Yamamoto for several useful discussions. We are also grateful

to H. Gies for pointing out the wrong placing of the derivative $\tilde{\partial}_{t}$ in Sec. III in the earlier manuscript. One of the authors (K.H.) is partially supported by Grant-in-Aid for Scientific Research on Priority Area, Number of Area 763, "Dynamics of Strings and Fields," from the Ministry of Education, Culture, Sports, Science and Technology, Japan.

\section{APPENDIX A: IR CUTOFF FUNCTION WITH FINITE $n$}

In this Appendix, we give a useful formula involving the IR cutoff function, and the dependence of the fixed points and the scaling dimensions of the RG equations on $n$.

\section{Evaluation of a typical loop integral}

A typical loop integral with the IR cutoff function $R_{\Lambda}^{(1)}\left(\boldsymbol{k}^{2}\right)$ which appears repeatedly in the derivation of our $\mathrm{RG}$ equations is

$$
I_{m, n} \equiv \int \frac{d^{3} k}{(2 \pi)^{3}} \tilde{\partial}_{t} \frac{\left(\boldsymbol{k}^{2}\right)^{m}}{A(P)-\frac{\boldsymbol{k}^{2}}{M}+2 R_{\Lambda}^{(1)}\left(\boldsymbol{k}^{2}\right)},
$$

where $A(P)$ is a Galilean invariant quantity,

$$
A(P) \equiv P^{0}-\frac{\boldsymbol{P}^{2}}{4 M}
$$

and $P^{\mu}=\left(P^{0}, \boldsymbol{P}\right)$ is the momentum flowing in (and out of) the diagram, $P=p_{1}+p_{2}=$ $p_{3}+p_{4}$, where $p_{1}$ and $p_{2}$ are initial momenta, and $p_{3}$ and $p_{4}$ are final momenta of the nucleons. The IR cutoff function is chosen as (3.5) for definiteness.

Switching to the polar coordinate, making the change of variable to $x=k / \Lambda$, and ex- 
panding the integrand with respect to $\tilde{A}(P) \equiv\left(M / \Lambda^{2}\right) A(P)$, we have

$$
\begin{aligned}
I_{m, n} & =-\frac{n}{\pi^{2}} M \Lambda^{2 m+1} \int_{0}^{\infty} d x x^{2 n+2 m+4} \exp \left(x^{2 n}\right)\left[\frac{\hat{R}(x)}{\tilde{A}(P)-x^{2}(1-\hat{R}(x))}\right]^{2} \\
& =-\frac{M \Lambda^{2 m+1}}{2 \pi^{2}}\left[\Gamma\left(1+\frac{2 m+1}{2 n}\right)+\tilde{A}(P) \Gamma\left(1+\frac{2 m-1}{2 n}\right)\left(2-2^{\frac{1-2 m}{2 n}}\right)+\mathcal{O}\left(\tilde{A}^{2}\right)\right],
\end{aligned}
$$

where $\hat{R}(x) \equiv\left(1-\exp \left(x^{2 n}\right)\right)^{-1}$ has been introduced. In the present paper, we disregard the terms of $\mathcal{O}\left(\tilde{A}^{2}\right)$ as higher orders.

We may decompose $A(P)$ into the following Galilean invariant quantities,

$$
A(P)=\left(p_{1}^{0}-\frac{\boldsymbol{p}_{1}^{2}}{2 M}\right)+\left(p_{2}^{0}-\frac{\boldsymbol{p}_{2}^{2}}{2 M}\right)+\frac{1}{4 M}\left(\boldsymbol{p}_{1}-\boldsymbol{p}_{2}\right)^{2} .
$$

Combining possible multiplicative momentum factors outside the integral, we can identify the contributions to the various operators in our theory space. For example, contributions from the first two terms renormalize a redundant operator and the last term does the operators which contain spatial derivatives.

\section{Dependence on the IR cutoff function}

The RG equations are derived and solved with a finite value of $n$. In this section, we show the $n$ dependence of the fixed points and the scaling dimensions.

The dependence on $n$ might give some useful information on the behavior of the present approximation. Note that if no approximation were made, the universal quantities such as the scaling dimension would not depend on how the RG transformation is defined. Weak dependence would suggest that the approximation is good. We will see shortly that the most of the fixed points as well as the scaling dimensions have very weak dependence. The fixed point with strong dependence on $n$ may be regarded as "spurious" and, for other fixed points, the approximation is very good.

In general, a sharp momentum cutoff gives a poor convergence and a smooth cutoff function is preferred[41]. In the present case, however, the dependence on $n$ is very weak and the use of a sharp cutoff is expected to be reasonable. In addition, it gives the simplest expressions. 
TABLE I: The dependence on $n$ of the fixed point $A$.

\begin{tabular}{lcc}
\hline \hline$n$ & location of the fixed point $\left(x^{\star}, y^{\star}, z^{\star}\right)$ & scaling dimensions \\
\hline 2 & $(-1.10326,-0.60467,0.60467)$ & $(-2,-1,1)$ \\
10 & $(-1.02722,-0.524991,0.524991)$ & $(-2,-1,1)$ \\
$10^{2}$ & $(-1.00287,-0.502587,0.502587)$ & $(-2,-1,1)$ \\
$10^{3}$ & $(-1.00029,-0.50026,0.50026)$ & $(-2,-1,1)$ \\
$10^{4}$ & $(-1.00003,-0.599926,0.500026)$ & $(-2,-1,1)$ \\
$\infty$ & $\left(-1,-\frac{1}{2}, \frac{1}{2}\right)$ & $(-2,-1,1)$ \\
\hline \hline
\end{tabular}

For a finite value of $n$, we find four fixed points including the trivial one. The dependence of the three nontrivial fixed points in the ${ }^{1} S_{0}$ channel is given in Tables 【, II] and III. (The dependence is similar for the ${ }^{3} S_{1}{ }^{3} D_{1}$ channel.) The last column shows the three eigenvalues of the linearized RG equations at the fixed point. Let us call these fixed points $A, B$, and $C$.

Note that, although we have an analytic expression for the RG equations for any $n$, the actual evaluation is done with the aid of a computer. We use Mathematica, but some numerical errors are not avoidable.

One can easily see that the fixed points $A$ and $B$ have little $n$ dependence (for $n \geq 10$ ), while the fixed point $C$ depends on $n$ strongly. We disregard the fixed point $C$. Note that even for the smallest integer value of $n, n=2(n=1$ does not satisfy the condition (3.4).), the scaling dimensions for the fixed point $A$ are the same while the fixed points $B$ and $C$ disappear there.

The fixed point $B$ has complex eigenvalues, though they have weak dependence on $n$. As discussed in the main text, the eigen-operators do not have the definite scaling properties there and we disregard it too in our discussion of the power counting. At the moment we know neither if it is just an artifact, nor, even if it is not, its physical implications. 
TABLE II: The dependence on $n$ of the fixed point $B$.

\begin{tabular}{ccc}
\hline \hline$n$ & location of the fixed point $\left(x^{\star}, y^{\star}, z^{\star}\right)$ & scaling dimensions \\
\hline 2 & Does not exist & Does not exist \\
10 & $(-9.85064,8.33328,-1.27074)$ & $(-0.79483 \pm 2.18601 i, 3)$ \\
$10^{2}$ & $(-9.03165,7.52438,-1.46746)$ & $(-0.502903 \pm 2.3961 i, 3)$ \\
$10^{3}$ & $(-9.00266,7.50188,-1.49663)$ & $(-0.50003 \pm 2.3979 i, 3)$ \\
$10^{4}$ & $(-9.00026,7.50018,-1.49966)$ & $(-0.494178 \pm 2.4005 i, 3.00465)$ \\
$\infty$ & $\left(-9, \frac{15}{2},-\frac{3}{2}\right)$ & $\left(-\frac{1}{2} \pm i \frac{\sqrt{23}}{2}, 3\right)$ \\
\hline \hline
\end{tabular}

TABLE III: The dependence on $n$ of the fixed point $C$.

\begin{tabular}{lcc}
\hline \hline$n$ & location of the fixed point $\left(x^{\star}, y^{\star}, z^{\star}\right)$ & scaling dimensions \\
\hline 2 & Does not exist & Does not exist \\
10 & $(-71.9608,77.804,-5.93769)$ & $(-62.929,0.874861,3)$ \\
$10^{2}$ & $(-6226.88,6961.68,-702.804)$ & $(-6202.06,0.99871,3)$ \\
$10^{3}$ & $(-609432,681363,-71583.4)$ & $(-609248,0.999984,3.81652)$ \\
$10^{4}$ & $\left(-6.08078 \times 10^{7}, 6.79826 \times 10^{7},-7.17129 \times 10^{6}\right)$ & $\left(-6.0806 \times 10^{7}, 1,3\right)$ \\
$\infty$ & Does not exist & Does not exist \\
\hline \hline
\end{tabular}

APPENDIX B: EXPLICIT DERIVATION OF RG EQUATIONS FROM THE AMPLITUDES

In this Appendix, we consider the (off-shell) two-nucleon amplitudes in the ${ }^{3} S_{1}-{ }^{3} D_{1}$ channel in order to derive the RG equations. (The amplitude in the ${ }^{1} S_{0}$ channel is given in Ref. [27].) The amplitudes take a matrix form due to the presence of the ${ }^{3} S_{1}-{ }^{3} D_{1}$ mixing and satisfy the following Lippmann-Schwinger equation in the center-of-mass frame,

$$
\begin{aligned}
-i \mathcal{A}_{i j}\left(p_{0}, \boldsymbol{p}_{2}^{2}, \boldsymbol{p}_{1}^{2}\right)= & -i V_{i j}\left(p_{0}, \boldsymbol{p}_{2}^{2}, \boldsymbol{p}_{1}^{2}\right) \\
& +\int \frac{d^{3} k}{(2 \pi)^{3}}\left(-i V_{i k}\left(p_{0}, \boldsymbol{p}_{2}^{2}, \boldsymbol{k}^{2}\right)\right) \frac{i}{p^{0}-\frac{\boldsymbol{k}^{2}}{2 M}+i \epsilon}\left(-i \mathcal{A}_{k j}\left(p_{0}, \boldsymbol{k}^{2}, \boldsymbol{p}_{1}^{2}\right)\right),
\end{aligned}
$$

where the subscript labels the partial waves, $i, j, k=\{S, D\}$, and $V_{i j}$ stands for the vertex in momentum space. The momentum integral is taken over $0 \leq k<\Lambda$. 
We take the averaged action (4.1) as our action, and write the vertex $V_{i j}$ to order $\mathcal{O}\left(p^{2}\right)$,

$$
\begin{aligned}
& V_{S S}=C_{0}^{(T)}+4 C_{2}^{(T)}\left(\boldsymbol{p}_{1}^{2}+\boldsymbol{p}_{2}^{2}\right)-2 B^{(T)}\left(p^{0}-\frac{\boldsymbol{p}_{1}^{2}+\boldsymbol{p}_{2}^{2}}{2 M}\right), \\
& V_{S D}=\frac{4 \sqrt{2}}{3} C_{2}^{(S D)} \boldsymbol{p}_{1}^{2}, \quad V_{D S}=\frac{4 \sqrt{2}}{3} C_{2}^{(S D)} \boldsymbol{p}_{2}^{2}, \quad V_{D D}=0,
\end{aligned}
$$

where $p^{0}$ is the center-of-mass energy, $\boldsymbol{p}_{1}$ and $\boldsymbol{p}_{2}$ are the relative momenta in the initial and final states respectively. We here suppress the spin and isospin dependence. See the appendix in Ref. 20] for a useful technique.

We consider the following ansatz,

$$
\begin{aligned}
& \mathcal{A}_{S S}\left(p^{0}, \boldsymbol{p}_{2}^{2}, \boldsymbol{p}_{1}^{2}\right)=T_{1}\left(p_{0}\right)+T_{2}\left(p_{0}\right)\left(\boldsymbol{p}_{1}^{2}+\boldsymbol{p}_{2}^{2}\right)+T_{3}\left(p_{0}\right) \boldsymbol{p}_{1}^{2} \boldsymbol{p}_{2}^{2}, \\
& \mathcal{A}_{S D}\left(p^{0}, \boldsymbol{p}_{2}^{2}, \boldsymbol{p}_{1}^{2}\right)=T_{4}\left(p_{0}\right) \boldsymbol{p}_{1}^{2}+T_{5}\left(p_{0}\right) \boldsymbol{p}_{1}^{2} \boldsymbol{p}_{2}^{2}, \\
& \mathcal{A}_{D S}\left(p_{0}, \boldsymbol{p}_{2}^{2}, \boldsymbol{p}_{1}^{2}\right)=T_{6}\left(p_{0}\right) \boldsymbol{p}_{2}^{2}+T_{7}\left(p_{0}\right) \boldsymbol{p}_{1}^{2} \boldsymbol{p}_{2}^{2}, \\
& \mathcal{A}_{D D}\left(p_{0}, \boldsymbol{p}_{2}^{2}, \boldsymbol{p}_{1}^{2}\right)=T_{8}\left(p_{0}\right) \boldsymbol{p}_{1}^{2} \boldsymbol{p}_{2}^{2},
\end{aligned}
$$

and obtain the solution,

$$
\begin{aligned}
& T_{1}=\frac{1}{D}\left[\left(C_{0}^{(T)}-2 B^{(T)} p^{0}\right)+\left\{\left(4 C_{2}^{(T)}+\frac{B^{(T)}}{M}\right)^{2}+\frac{32}{9}\left(C_{2}^{(S D)}\right)^{2}\right\} I_{2}\right] \\
& T_{2}=\frac{1}{D}\left(4 C_{2}^{(T)}+\frac{B^{(T)}}{M}\right)\left[1-\left(4 C_{2}^{(T)}+\frac{B^{(T)}}{M}\right) I_{1}\right] \\
& T_{3}=\frac{1}{D}\left(4 C_{2}^{(T)}+\frac{B^{(T)}}{M}\right)^{2} I_{0} \\
& T_{4}=T_{6}=\frac{1}{D} \frac{4 \sqrt{2}}{3} C_{2}^{(S D)}\left(1-\left(4 C_{2}^{(T)}+\frac{B^{(T)}}{M}\right) I_{1}\right) \\
& T_{5}=T_{7}=\frac{1}{D} \frac{4 \sqrt{2}}{3} C_{2}^{(S D)}\left(4 C_{2}^{(T)}+\frac{B^{(T)}}{M}\right) I_{0}, \\
& T_{8}=\frac{32}{9} \frac{1}{D}\left(C_{2}^{(S D)}\right)^{2} I_{0}
\end{aligned}
$$

with

$$
\begin{aligned}
D= & 1-\left(C_{0}^{(T)}-2 B^{(T)} p^{0}\right) I_{0}-2\left(4 C_{2}^{(T)}+\frac{B^{(T)}}{M}\right) I_{1}+\left(4 C_{2}^{(T)}+\frac{B^{(T)}}{M}\right)^{2} I_{1}^{2} \\
& -\left(\left(4 C_{2}^{(T)}+\frac{B^{(T)}}{M}\right)^{2}+\frac{32}{9}\left(C_{2}^{(S D)}\right)^{2}\right) I_{0} I_{2},
\end{aligned}
$$

where $I_{n}$ are integrals defined by

$$
I_{n}=-\frac{M}{2 \pi^{2}} \int^{\Lambda} d k \frac{k^{2 n+2}}{k^{2}+\mu^{2}}, \quad \mu=\sqrt{-M p^{0}-i \epsilon} .
$$


The RG equations for the couplings are obtained by requiring that the inverse of the offshell amplitudes $\left(\mathcal{A}_{S S}, \mathcal{A}_{S D}\right.$ in this case) are independent of the cutoff $\Lambda$, in the expansion in powers of $\boldsymbol{p}_{1}^{2}, \boldsymbol{p}_{2}^{2}$, and $\mu^{2}$. We introduce dimensionless coupling constants as (4.12).

The resulting $\mathrm{RG}$ equations are written as

$$
\begin{aligned}
\Lambda \frac{d X}{d \Lambda} & =\frac{1}{X}(X-1)\left(Y+3 X^{2}\right) \\
\Lambda \frac{d Y}{d \Lambda} & =\frac{Y}{X^{2}}\left(6 X^{3}-5 X^{2}+2 X Y-Y\right) \\
\Lambda \frac{d Z}{d \Lambda} & =\frac{1}{X^{2}}\left(6 X^{3} Z-3 X^{2} Z+2 X Y Z+Y^{2}\right) \\
\Lambda \frac{d w^{\prime}}{d \Lambda} & =\frac{1}{X}\left(3 X^{2} w^{\prime}+Y w^{\prime}\right)
\end{aligned}
$$

where we have introduced $X=1+\left(y^{\prime}+z^{\prime}\right) / 3, Y=x^{\prime}-\left(\left(y^{\prime}+z^{\prime}\right)^{2}+2 w^{\prime 2}\right) / 5$, and $Z=$ $2 y^{\prime}+\left(\left(y^{\prime}+z^{\prime}\right)^{2}-2 w^{\prime 2}\right) / 3$. Compare them with those obtained in Ref. [27] for the ${ }^{1} S_{0}$ channel. They are identical if one sets $w^{\prime}=0$.

Although they look complicated and very different from Eqs. (4.13), when expanded in couplings, they agree with Eqs. (4.13) to the second order.

These RG equations have only two fixed points (as far as Mathematica can find); a trivial fixed point $\left(X^{\star}, Y^{\star}, Z^{\star}, w^{\star}\right)=(1,0,0,0)$ and a nontrivial fixed point $\left(X^{\star}, Y^{\star}, Z^{\star}, w^{\star}\right)=$ $(1,-1,-1,0)$ which corresponds to $\left(x^{\prime \star}, y^{\prime \star}, z^{\prime \star}, w^{\prime \star}\right)=(-1,-1 / 2,1 / 2,0)$. The locations of these fixed points are the same as those obtained from Eqs. (4.13).

Substituting

$$
x^{\prime}=x^{\star \star}+\delta x^{\prime}, \quad y^{\prime}=y^{\prime \star}+\delta y^{\prime}, \quad z^{\prime}=z^{\prime \star}+\delta z^{\prime}, \quad w^{\prime}=w^{\prime \star}+\delta w^{\prime},
$$

into (B8), we obtain the linearized RG equations at the nontrivial fixed point,

$$
\frac{d}{d t}\left(\begin{array}{c}
\delta x^{\prime} \\
\delta y^{\prime} \\
\delta z^{\prime} \\
\delta w^{\prime}
\end{array}\right)=\left(\begin{array}{cccc}
1 & 2 & 2 & 0 \\
2 & \frac{2}{3} & \frac{5}{3} & 0 \\
-2 & -\frac{8}{3} & -\frac{11}{3} & 0 \\
0 & 0 & 0 & -2
\end{array}\right)\left(\begin{array}{c}
\delta x^{\prime} \\
\delta y^{\prime} \\
\delta z^{\prime} \\
\delta w^{\prime}
\end{array}\right)
$$

where we have introduced $t=\ln \left(\Lambda_{0} / \Lambda\right)$. 
These equations can be solved easily,

$$
\begin{aligned}
\left(\begin{array}{c}
\delta x^{\prime} \\
\delta y^{\prime} \\
\delta z^{\prime} \\
\delta w^{\prime}
\end{array}\right)=a_{1}^{\prime}\left(\begin{array}{c}
2 \\
1 \\
-4 \\
0
\end{array}\right)\left(\frac{\Lambda}{\Lambda_{0}}\right)^{2}+a_{2}^{\prime}\left(\begin{array}{l}
0 \\
0 \\
0 \\
1
\end{array}\right)\left(\frac{\Lambda}{\Lambda_{0}}\right)^{2} \\
+b^{\prime}\left(\begin{array}{c}
0 \\
-1 \\
1 \\
0
\end{array}\right)\left(\frac{\Lambda}{\Lambda_{0}}\right)+c^{\prime}\left(\begin{array}{c}
1 \\
1 \\
-1 \\
0
\end{array}\right)\left(\frac{\Lambda_{0}}{\Lambda}\right)
\end{aligned}
$$

where $a_{1}^{\prime}, a_{2}^{\prime}, b^{\prime}$, and $c^{\prime}$ are dimensionless constants. This corresponds to Eq. (4.24).

We obtain the renormalized off-shell amplitudes near the nontrivial fixed point to the second order in powers of $\boldsymbol{p}^{2}$ and $p^{0}$ by substituting the solutions of linearized RG equations, for example,

$$
\left.\mathcal{A}_{S S}^{-1}\left(p_{0}, \boldsymbol{p}^{\prime 2}, \boldsymbol{p}^{2}\right)\right|_{*}=-\frac{M}{4 \pi}\left[\frac{2}{\pi} c^{\prime} \Lambda_{0}-\frac{4}{\pi} b^{\prime}\left(\frac{M p_{0}}{\Lambda_{0}}\right)-i \sqrt{M p_{0}}\right]+\cdots,
$$

where the ellipsis denotes terms of higher orders of $\boldsymbol{p}^{2} / \Lambda_{0}$ and $p^{0} / \Lambda_{0}$. We notice that $a_{1}^{\prime}$ and $a_{2}^{\prime}$ do not contribute to the inverse of the amplitude to this order. The existence of such eigenvectors whose coefficients do not show up in the on-shell amplitudes is related to the equivalence theorem considered in the previous paper 27]. Actually, the equivalence relation (3.21) of Ref. 27] gives "trajectories" in the space of couplings, and the tangential direction at the nontrivial fixed point is the direction of the eigenvector whose coefficient does not appear in the physical amplitude. This would suggest that all the "momentum-dependent perturbations" discussed in Ref. [26] form "surfaces" in the space of couplings near the fixed point on which any two points are related by some equivalence relation.

[1] V. G. J. Stoks, R. A. M. Klomp, C. P. F. Terheggen, and J. J. de Swart, Phys. Rev. C49, 2950 (1994), nucl-th/9406039.

[2] R. B. Wiringa, V. G. J. Stoks, and R. Schiavilla, Phys. Rev. C51, 38 (1995), nucl-th/9408016.

[3] R. Machleidt, F. Sammarruca, and Y. Song, Phys. Rev. C53, 1483 (1996), nucl-th/9510023.

[4] R. Machleidt, Phys. Rev. C63, 024001 (2001), nucl-th/0006014. 
[5] S. Weinberg, Phys. Lett. B251, 288 (1990).

[6] S. Weinberg, Nucl. Phys. B363, 3 (1991).

[7] S. Weinberg, Physica A96, 327 (1979).

[8] G. P. Lepage (1989), hep-ph/0506330.

[9] J. Polchinski (1992), hep-th/9210046.

[10] H. Georgi, Ann. Rev. Nucl. Part. Sci. 43, 209 (1993).

[11] D. B. Kaplan (1995), nucl-th/9506035.

[12] A. V. Manohar (1996), hep-ph/9606222.

[13] J. Gasser and H. Leutwyler, Ann. Phys. 158, 142 (1984).

[14] J. Gasser and H. Leutwyler, Nucl. Phys. B250, 465 (1985).

[15] A. Manohar and H. Georgi, Nucl. Phys. B234, 189 (1984).

[16] D. B. Kaplan, M. J. Savage, and M. B. Wise, Nucl. Phys. B478, 629 (1996), nucl-th/9605002.

[17] A. Nogga, R. G. E. Timmermans, and U. van Kolck, Phys. Rev. C72, 054006 (2005), nuclth/0506005.

[18] D. B. Kaplan, M. J. Savage, and M. B. Wise, Phys. Lett. B424, 390 (1998), nucl-th/9801034.

[19] D. B. Kaplan, M. J. Savage, and M. B. Wise, Nucl. Phys. B534, 329 (1998), nucl-th/9802075.

[20] S. Fleming, T. Mehen, and I. W. Stewart, Nucl. Phys. A677, 313 (2000), nucl-th/9911001.

[21] S. R. Beane, P. F. Bedaque, M. J. Savage, and U. van Kolck, Nucl. Phys. A700, 377 (2002), nucl-th/0104030.

[22] M. C. Birse (2005), nucl-th/0507077.

[23] D. B. Kaplan (2005), nucl-th/0510023.

[24] K. G. Wilson and J. B. Kogut, Phys. Rept. 12, 75 (1974).

[25] K. G. Wilson, Rev. Mod. Phys. 47, 773 (1975).

[26] M. C. Birse, J. A. McGovern, and K. G. Richardson, Phys. Lett. B464, 169 (1999), hep$\mathrm{ph} / 9807302$.

[27] K. Harada, K. Inoue, and H. Kubo, Phys. Lett. B636, 305 (2006), nucl-th/0511020.

[28] C. Wetterich, Nucl. Phys. B352, 529 (1991).

[29] C. Wetterich, Phys. Lett. B301, 90 (1993).

[30] J. Berges, N. Tetradis, and C. Wetterich, Phys. Rept. 363, 223 (2002), hep-ph/0005122.

[31] K. Harada, H. Kubo, and Y. Yamamoto, in preparation.

[32] U. van Kolck, Nucl. Phys. A645, 273 (1999), nucl-th/9808007. 
[33] T. Mehen and I. W. Stewart, Phys. Lett. B445, 378 (1999), nucl-th/9809071.

[34] T. Mehen and I. W. Stewart, Phys. Rev. C59, 2365 (1999), nucl-th/9809095.

[35] D. B. Kaplan and M. J. Savage, Phys. Lett. B365, 244 (1996), hep-ph/9509371.

[36] D. B. Kaplan and A. V. Manohar, Phys. Rev. C56, 76 (1997), nucl-th/9612021.

[37] T. Mehen, I. W. Stewart, and M. B. Wise, Phys. Rev. Lett. 83, 931 (1999), hep-ph/9902370.

[38] D. B. Kaplan, Nucl. Phys. B494, 471 (1997), nucl-th/9610052.

[39] P. F. Bedaque and U. van Kolck, Phys. Lett. B428, 221 (1998), nucl-th/9710073.

[40] P. F. Bedaque, H. W. Hammer, and U. van Kolck, Phys. Rev. C58, 641 (1998), nuclth/9802057.

[41] D. F. Litim, JHEP 11, 059 (2001), hep-th/0111159. 